\title{
Oral Administration to Nursing Women of Lactobacillus fermentum CECT5716 Prevents Lactational Mastitis Development: A Randomized Controlled Trial
}

\author{
José A. Hurtado,' Jose A. Maldonado-Lobón,' M. Paz Díaz-Ropero, Katherine Flores-Rojas, \\ José Uberos, José L. Leante, Laura Affumicato, María Luz Couce, José M. Garrido, \\ Mónica Olivares, ${ }^{2}$ Juristo Fonollá; ${ }^{2}$ and the PROLAC Group*
}

\begin{abstract}
Objective: The objective of this study is to evaluate the preventive effect of oral administration of Lactobacillus fermentum CECT5716 on mastitis incidence in lactating women.

Methods: A randomized double-blinded controlled trial that included 625 women was conducted. Women who received preventive dose of antibiotic in the context of delivery were recruited 1-6 days after childbirth and randomly assigned to a group. Probiotic group received 1 capsule/day containing L. fermentum $3 \times 10^{9} \mathrm{CFU}$, control group received 1 placebo capsule/day containing maltodextrin. The intervention period was 16 weeks. The primary outcome of the study was the incidence of clinical mastitis defined as at least two out of the three breast symptoms (pain, redness, and lump) and at least one of fever or flu-like symptoms (shivering, hot sweats, or aches).

Results: Two hundred ninety-one women completed 16 weeks of treatment. Sixteen women in the probiotic group developed mastitis versus 30 women in the control group (odds ratio $=0.531 ; p=0.058$ ). Incidence rate of mastitis in the probiotic group was significantly lower than that in the control group (IR $=0.130$ in the probiotic group versus $\mathrm{IR}=0.263$ in the control group; $p=0.021$ ). Therefore, the oral administration of L. fermentum CECT5716 during lactation decreased by $51 \%$ the incidence rate of clinical mastitis. Staphylococcus spp. load at the end of intervention was significantly lower in breast milk of women in the probiotic group than in breast milk of women in the control group $(p=0.025)$.

Conclusion: Consumption of the probiotic strain L. fermentum CECT5716 might be used during breastfeeding as an efficient strategy to prevent development of lactational mastitis in women.
\end{abstract}

Trial registration: NCT02203877.

Keywords: mastitis, prevention, probiotics

${ }^{1}$ Department of Neonatology, Hospital Materno-Infantil del CHU, Granada, Spain.

${ }^{2}$ Research Department, Biosearch Life, Granada, Spain.

${ }^{3}$ Department of Metabolism and Pediatrics Research, Hospital Reina Sofía, Córdoba, Spain.

${ }^{4}$ Department of Pediatrics, School of Medicine, University of Granada, Spain.

${ }^{5}$ Department of Neonatology, Hospital G.U. Santa Lucía, Cartagena, Murcia, Spain.

${ }^{6}$ Department of Neonatology, Hospital R.U., Málaga, Spain.

${ }^{7}$ Department of Neonatology, Hospital C.H.U., Santiago de Compostela, La Coruña, Spain.

${ }^{8}$ Department of Pediatrics, Hospital U., Salamanca, Spain.

* Members of the PROLAC Group are listed under Acknowledgments at the end of the article.

(C) José A. Hurtado et al., 2017; Published by Mary Ann Liebert, Inc. This Open Access article is distributed under the terms of the Creative Commons License (http://creativecommons.org/licenses/by/4.0), which permits unrestricted use, distribution, and reproduction in any medium, provided the original work is properly credited. 


\section{Introduction}

$\mathbf{H}$ UMAN MICROBIOTA PLAYS an important and increasingly recognized role in human health. Dysbiosis in microbiota of different locations of human organism has been related to diseases such as inflammatory bowel disease, diabetes, obesity, allergy, vaginal infections, atopic dermatitis, and tooth decay. ${ }^{1}$ Interestingly, probiotics are showing beneficial effects on these diseases by restoring the balance of the microbiota. ${ }^{2,3}$ In 2003, the presence of a physiological microbiota was first described in human milk. ${ }^{4,5}$ Recent studies have revealed that dysbiosis in this specific microbiota is related to mastitis. ${ }^{6-8}$

Mastitis is an inflammatory condition of the breast usually associated with lactation. Dysbiosis in mastitis is characterized by proliferation of certain bacterial species such as Staphylococcus spp., which has been identified as one of the main bacterial groups related to mastitis. ${ }^{6,9-11}$ Data about the incidence of this problem are very variable probably because of differences in diagnosis criteria. WHO refers data of incidence highly variable that can reach $33 \%$. $^{9}$ During the past decade, different studies have demonstrated the capability of certain probiotic strains to balance the microbiota in human milk by reducing load of bacterial groups related to mastitis. $^{12-14}$ The decrease in bacterial load was related to a decrease in the severity of the disease. These studies have provided some evidence about the potential of probiotic bacteria to efficiently treat the problem of mastitis.

Lactobacillus fermentum CECT5716 is a probiotic strain isolated from breast milk of healthy woman. ${ }^{15}$ This strain has shown powerful anti-infectious activity probably related to its antibacterial activity and inmuno-enhancing activity. ${ }^{16-19}$ Regarding mastitis, two different studies have demonstrated that L. fermentum CECT5716 significantly improves mastitis condition by decreasing Staphylococcus spp. load in breast milk. ${ }^{13,14}$ Staphylococcus spp. is the main causal agent of mastitis and it is a risk factor for the disease. ${ }^{6,9,10}$ The capability of L. fermentum CECT5716 to reduce this important risk factor for mastitis encourages us to perform a clinical trial to evaluate the potential of this strain to prevent the development of lactational mastitis.

\section{Materials and Methods}

\section{Study design and protocol}

A randomized double-blinded placebo-controlled multicenter trial was performed. The inclusion criteria were as follows: healthy women between 18 and 45 years with development of normal pregnancy, childbirth took place 16 days before recruitment, birth between 37 and 42 weeks of gestation, women who had received preventive antibiotic treatment between 48 hours before and 48 hours after childbirth (one dose was sufficient for inclusion regardless of the type of antibiotic), and women with firm intention to breastfeed their children for at least 16 weeks. Exclusion criteria during the study were mammary pathologies or children's pathologies that hinder or preclude breastfeeding and low expectation of adherence to the study protocol. Written informed consent was obtained from the women.

Sample size was estimated based on the effect on the main outcome of the study and the incidence of mastitis. Based on previous data about mastitis incidence, the study was designed to exhibit sufficient power $(80 \%)$ to detect a difference between groups of $40 \%$ in the incidence rate of mastitis after treatment with a 0.05 significance level (Software $R$ version 2.12.2). The number of women necessary was 258 per group, total sample size was increased up to 625 women to compensate dropouts.

Women were recruited and distributed into two study groups, according to a randomization generated by a computer program ( $\mathrm{R}$ version 2.12.2). Probiotic group received L. fermentum CECT5716 for 16 weeks at doses of $3 \times 10^{9}$ $\mathrm{CFU} /$ day. Control group received a placebo of maltodextrin.

L. fermentum CECT5716 was provided by Biosearch Life. Capsules containing $3 \times 10^{9} \mathrm{CFU} /$ capsule of L. fermentum CECT5716 or maltodextrin were prepared by Biofabri S.L. (A Relva s/n 36400 O Porriño, Pontevedra, Spain).

Women consumed one capsule per day containing probiotic (probiotic group) or maltodextrin (control group). Probiotic and placebo were provided in identical gelatin capsules packaged in identical plastic tubes labeled in plain white with a code number that referred to the manufacturing batch. As stability of probiotic bacteria is dependent on temperature of conservation, capsules were kept at $4^{\circ} \mathrm{C}$ throughout the study. The concentration of viable Lactobacillus species in the probiotic capsules was stable throughout the study $\left(2.9 \times 10^{9} \mathrm{CFU} /\right.$ capsule at the end of the intervention).

Twelve hospitals from different regions of Spain collaborated in the study. The study was performed in accordance with the Helsinki declaration, and the protocol was approved by the Regional Ethics Committee of the Sistema Andaluz de Salud based in Seville (Spain) for hospital in Andalusia region and for local Ethics Committees for the rest of hospitals. The trial was registered in the U.S. Library of Medicine (www.clinicaltrial.gov) with the number NCT02203877.

\section{Study outcomes}

The primary outcome of the trial was the incidence rate of mastitis during the first 4 months of breastfeeding. Mastitis was defined as reported by Amir et al. ${ }^{20}$ : at least two out of the three breast symptoms (pain, redness, and lump) and at least one of fever or flu-like symptoms (shivering, hot sweats, or aches).

Secondary outcomes were microbiota of breast milk at the end of intervention and in mastitis events, monthly questionnaire on evaluation of breast pain, and inflammatory markers in breast milk at the end of intervention and in mastitis events.

\section{Data collection}

For mastitis diagnosis, the presence of local symptoms in breast (pain, redness, and lump) and systemic symptoms (fever, shivering, hot sweats, or aches) was recorded. Women were contacted monthly by phone and asked about mastitis symptoms and asked to score their breast pain from 0 (no pain) to 10 (extremely painful). In case of mastitis symptoms, diagnosis was confirmed by a corresponding medical doctor or midwife. Data about use of antibiotic, analgesic, and topic treatment for nipple and for breast symptoms were also collected.

As changes in diet might influence the results on main outcomes of the study, this variable was controlled by a Food Frequency Questionnaire. The questionnaire was completed by the women at the beginning and at the end of the intervention. Compliance of women was evaluated by collecting the remaining capsules at the end of intervention. 
Breast milk samples were collected at the beginning and at the end of intervention. In case of mastitis event, breast milk samples were also collected. For breast milk sample collection, nipple and mammary areola were cleaned with soap and water and an antibacterial (chlorhexidine) solution was applied. Breast milk samples were obtained by manual expression and collected in sterile tubes after discarding the first drops of milk. Samples were preserved at $-20^{\circ} \mathrm{C}$ and processed within 1 month.

\section{Breast milk bacteria quantification}

To estimate the concentration of total bacteria in breast milk, appropriate dilutions of samples in buffered peptonized water (bioMérieux SA, Marcy de Marcy l'Etoile, France) were spread in quadruplicate onto plates of plate count agar (PCA) and Wilkins-Chalgren anaerobe agar (WCA) (Oxoid, Basingstoke, United Kingdom). The cultures were incubated in aerobic (PCA) and anaerobic (WCA) conditions (AnaeroGen; Oxoid, Basingstoke, United Kingdom) at $37^{\circ} \mathrm{C}$ for 48 hours. After the incubation, the colonies grown on the culture media were counted, and the number of viable microorganisms per milliliter of milk (CFU/mL) was calculated.

Staphylococci, Streptococci, and Lactobacilli counts were measured by quantitative PCR following the method described in Maldonado-Lobón et al., ${ }^{14}$ except for the common thermal profile applied for amplification, using this time $95^{\circ} \mathrm{C}$ for 5 minutes followed by 40 cycles of $95^{\circ} \mathrm{C}$ for 30 seconds and $65^{\circ} \mathrm{C}$ for 60 seconds, and a final melting curve from $55^{\circ} \mathrm{C}$ to $95^{\circ} \mathrm{C}$.

For L. fermentum detection, breast milk samples (250$500 \mu \mathrm{L}$ ) were used as inocula for Lactobacillus spp. enrichment cultures performed in three different broth media (15 mL): M.R.S. (Oxoid) supplemented with vancomycin (100 $\mu \mathrm{g} / \mathrm{mL}$; Sigma-Aldrich); M.R.S. pH adjusted to 4.5 and Rogosa broth. After 24-48 hours of anoxic incubation at $37^{\circ} \mathrm{C}, 100 \mu \mathrm{L}$ of liquid cultures was spread on the equivalent corresponding agar medium, except for cultures performed in M.R.S. pH 4.5, which were spread on LAMVAB agar medium. Petri dishes were incubated for 48 hours at $37^{\circ} \mathrm{C}$ under anoxic atmosphere (AnaeroGen, Oxoid). Colonies with Lactobacillus characteristic morphology were selected and identified by MALDI-TOF mass spectrometry (Bruker Biotyper). In brief, a small amount of biomass was transferred from selected colonies to a 96 -spot plate, then $1 \mu \mathrm{L}$ of matrix solution (2-cyano-3-(4-hydroxyphenyl) acrylic acid) was added over every sample and plate was dried at room temperature for 10-15 minutes. Analytical runs included L. fermentum CECT 5716 as positive control and accurate identification at species level was assumed when control and samples showed a score greater or equal to 2.2 .

\section{Interleukin-8 quantification in breast milk}

Interleukin (IL)-8 concentrations were measured in breast milk samples by ELISA quantification kits following the manufacturer's instructions (Bethyl, Montgomery, TX and Diaclone, France).

\section{Statistical analysis}

For continuous outcomes, statistical tests for differences in the effect of treatment per visit were performed using parametric ( $t$ test, if normality assumption is met) and nonparametric (Mann-Whitney $U$ and bootstrap confidence intervals, if normality assumption is not met) tests.

For the mother's primary outcome, diagnosis of mastitis, the total number of events from the study period was counted and the incidence rates were obtained. For categorical outcomes per visit, chi-square test for binary or categorical responses was used. Finally, a more robust and accurate analysis through statistical modeling was performed to determine the effect of the treatment along the study adjusted by relevant covariates gathered in the study.

The models applied to the data were Linear Mixed Models for continuous data when the residuals were normally distributed, Ordinal Mixed Models when the data recorded were related to categories of number of frequencies a day/week/ month or any type of variable with increasing or decreasing order for the defined categories, and Logistic Mixed Models when the outcome to be analyzed was binary responses. A Poisson regression model was used to examine differences in the number of events observed of diagnosed mastitis.

The tests were performed at the two-sided 5\% significance level, and $95 \%$ confidence intervals were obtained for the estimates.

The statistical software used to perform the analysis was SPSS version 19 and $R$ version 3.1.

\section{Results \\ Participants}

Recruitment was started in August 2013 and ended in April 2015. The intervention was completed in July 2015.

A flow chart of the participants in the study is shown in Figure 1. Six hundred twenty-five women were recruited for the study. Women were randomized and received the treatment (322 in control group and 303 in probiotic group). Three hundred thirty-four women discontinued the treatment because of causes reported in Figure 1. Four hundred twentyfive women completed 1 month of intervention (221 in control group and 203 in probiotic group), 359 completed 2 months of intervention (186 in control group and 173 in probiotic group), and 309 completed 3 months of intervention (165 in control group and 144 in probiotic group). Finally, 291 women (152 in the control group and 139 in probiotic group) finalized the intervention of 16 weeks. Data of these 291 women were included in the analysis of the mother's primary outcomes and incidence rates of mastitis during the first 4 months of breastfeeding. For evaluation of symptoms at each month, all data available were taken into account in the analysis.

No significant differences were detected between the baseline characteristics of women in the two groups (Table 1). Level of education and living area (city center, neighborhood, close to city, and rural area) of women were similar between groups ( $p=0.446$ and $p=0.216$, respectively).

Participants were asked to fill in a questionnaire regarding their diets at recruitment and at the end of the study period. Although some differences were detected between baseline and final time, no significant differences were detected between both groups (data not shown). Therefore, the significant effects observed for outcomes cannot be attributed to participant's different dietary habits. 
FIG. 1. Flowchart of participants. (1) Causes: mother's decision, perception of insufficient milk, mastitis. (2) Causes: gastrointestinal problems in infants, maternal rash.

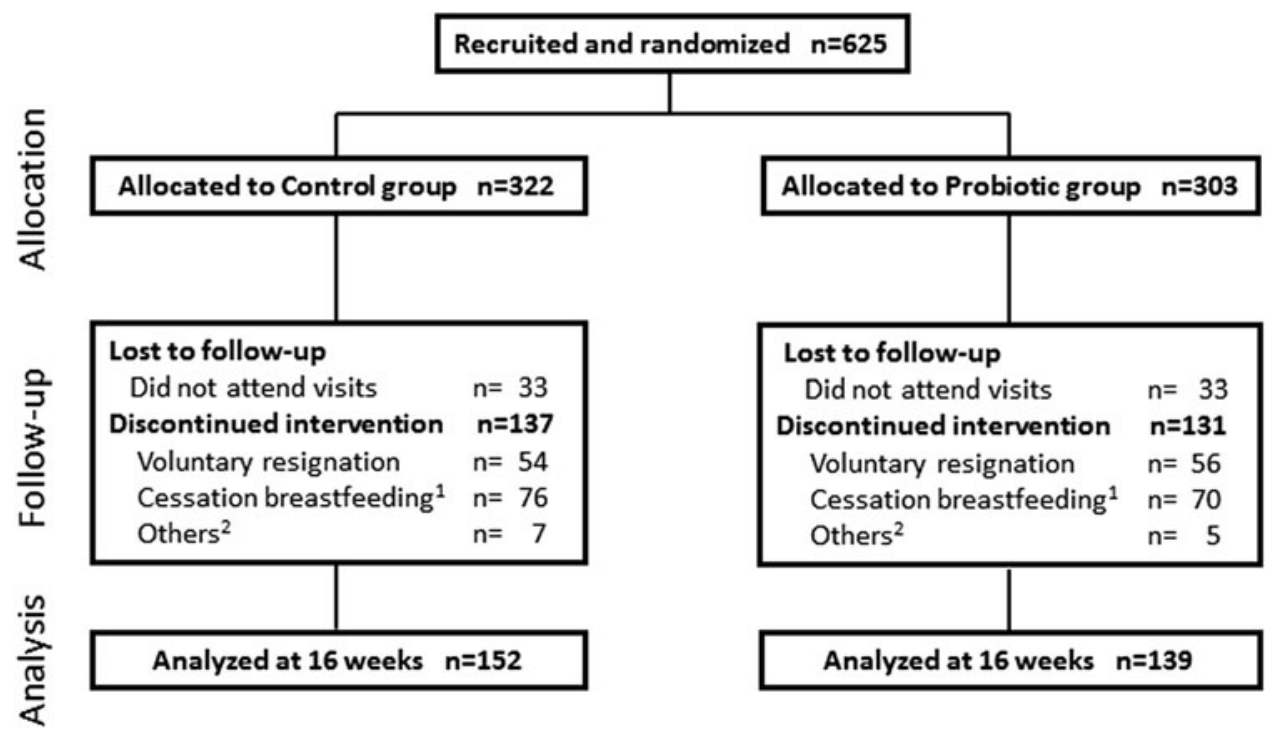

\section{Incidence of mastitis}

Sixteen women in the probiotic group suffered mastitis events in contrast to 30 in the control group. From events of diagnosed mastitis each month, the total number of events observed during the study for each group was built. A total of 58 events of mastitis were diagnosed in women of the study during intervention (incidence rate 0.199). Incidence rate of mastitis in probiotic group was significantly lower than that in the control group ( $I R=0.130$ in probiotic group versus $I R=0.263$ in control group; $p=0.021$ ) (Table 2). Therefore, the oral administration of L. fermentum CECT5716 during lactation decreased by $51 \%$ the incidence rate of clinical mastitis.

\section{Incidence of symptoms related to mastitis}

Data about the incidence rate of different symptoms related to mastitis were also analyzed. The values of incidence rates of different symptoms were lower in the probiotic group (range 16-42\% of decrease), reaching statistical significance for presence of heat zones in breast $(p=0.024)$. The most frequent symptom was breast pain. The distribution of the responses of breast pain during the first month of breastfeeding indicates higher percentage of women with breast pain in the control group than women in the probiotic group (43\% versus $33 \% ; p=0.033$ ). The odds of having breast pain in the probiotic group were significantly lower than odds of having breast pain in the control group $(\mathrm{OR}=0.65 ; 95 \% \mathrm{CI}$ 0.44-0.97). In particular, odds of breast pain in the control group are 1.5 times the odds of pain in the probiotic group. Percentage of women suffering from breast pain decreased with time, and in the fourth month only $6.5 \%$ of women in the probiotic group reported breast pain versus $9.2 \%$ in the control group ( $p=0.515)$. Regarding intensity of breast pain, significant overall decrease along the study was observed in both groups. Although means of values of pain intensity were low, a slight difference between groups was detected in pain intensity during the first month of breastfeeding ( 2.92 in the control group versus 2.55 in the probiotic group; $p=0.05)$.

\section{Bacterial counts}

Bacterial load was evaluated in milk samples of women at the end of the treatment and in case of mastitis event (Table 3). In the case of healthy women, lower level of Staphylococcus spp. was observed in breast milk of women of the probiotic group than in that of women in the control group $(-48 \% ; p=0.013)$. In case of breast milk samples collected in case of mastitis events, lower level of Staphylococcus spp. load was also observed in samples from

Table 1. Baseline Characteristics of the Participants of the Study

\begin{tabular}{lcrl}
\hline & Control group $(\mathrm{n}=322)$ & Probiotic group $(\mathrm{n}=303)$ & $\mathrm{p} /$ chi-squared \\
\hline Mother's age $($ mean $\pm \mathrm{SD})$, years & $32.19 \pm 4.8$ & $31.91 \pm 4.9$ & 0.487 \\
C-section, $n(\%)$ & $101(31.4)$ & $105(34.7)$ & 0.422 \\
Multiparas, $n(\%)$ & $133(41.3)$ & $113(37.3)$ & 0.777 \\
Previous mastitis events in multiparas, $n(\%)$ & $23(17.3)$ & $94(31.5)$ & 0.126 \\
Use of pacifier, $n(\%)$ & $98(30.5)$ & $164(54.3)$ & 0.862 \\
Mixed breastfeeding, $n(\%)$ & $159(49.4)$ & $90(26.4)$ & 0.419 \\
Sucking difficulties, $n(\%)$ & $94(29.2)$ & $9.09 \pm 2.6$ & 0.419 \\
Number of daily breastfeedings, mean $\pm \mathrm{SD}$ & $9.03 \pm 3.0$ & $34(11.2)$ & 0.655 \\
Smoker at recruitment, $n(\%)$ & $29(9.0)$ & 0.344 \\
\hline
\end{tabular}

${ }^{a}$ Number and percentage of infants who received infant formula as well as breast milk before starting the intervention. 
Table 2. Incidence of Mastitis During First 4 Months of Breastfeeding

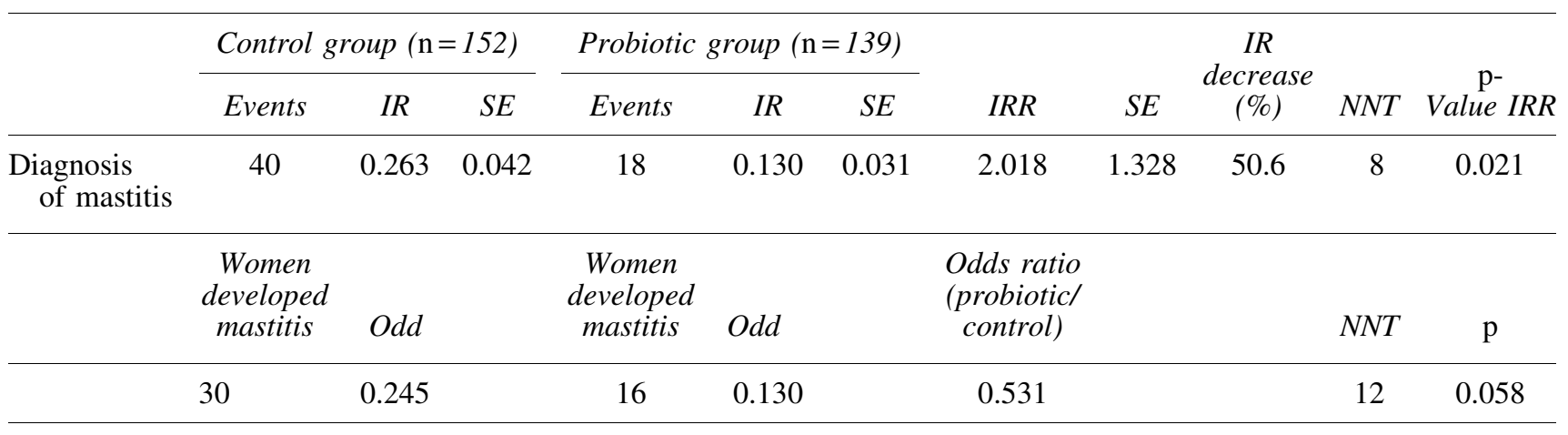

IR, incidence rate; IRR, incidence rate ratio; NNT, number of patients needed to treat; SE, standard error.

women of the probiotic group than in samples of women of the control group $(-58 \% ; p=0.065)$. Significant differences were found in the Streptococcus and Staphylococcus loads, having lower values in average in women without mastitis. Higher level of Staphylococcus or Streptococcus is associated with the risk of suffering from mastitis [odds ratio for Staphylococcus is 1.961 (95\% CI 1.272-3.025), $p=0.002$; odds ratio for Streptococcus is 1.687 (95\% CI 1.049-2.714), $p=0.031]$.

No significant differences were observed in Lactobacillus, total aerobes, and anaerobes counts.

Breast milk samples at the end of intervention were cultured in specific selective culture medium to favor the growth of Lactobacillus species. The specie L. fermentum was detected in breast milk in $23.6 \%$ of women in the probiotic group and in $14.6 \%$ of women in the control group.

\section{Inflammatory marker in breast milk (IL-8)}

IL-8 concentration in milk was measured in samples of women at the end of the treatment and in case of mastitis event (Table 3). No significant differences were detected in IL-8 level between probiotic and control groups in the case of healthy women. However, IL-8 level was significantly higher in breast milk of women suffering from mastitis than in breast milk of healthy women. In these cases of mastitis events, women receiving probiotic strain showed significantly lower concentration of IL-8 in breast milk than that in breast milk in women of the control group ( $p=0.037)$.

A general association of Staphylococuss with IL-8 was detected, which means that with per unit increase of bacterial load, the concentration of IL- 8 increases significantly in $0.147 \mathrm{U}(p<0.001)$. The odds of having mastitis increase five times for unit increase of IL-8 [odds ratio $=5.502(95 \% \mathrm{CI}$ 2.904-10.425), $p=0.000]$.

\section{Pharmacological treatments}

In the probiotic group, four women $(2.9 \%)$ received antibiotic treatment in contrast to eight women $(5.3 \%)$ in the control group; however, difference was not statistically significant $(p=0.308)$. Analgesic consumption was lower in the

Table 3. Bacterial Counts and Concentration of Interleukin-8 in Breast Milk in Healthy Women at the End of Intervention (4 Months) and in Case of Mastitis Event

\begin{tabular}{|c|c|c|c|c|c|c|c|c|}
\hline & $\begin{array}{c}\text { Group } \\
\text { (control group, } \\
\mathrm{n}=123 ; \\
\text { probiotic } \\
\text { group } \mathrm{n}=126)\end{array}$ & Healthy & $\begin{array}{c}95 \% \\
\text { Confidence } \\
\text { interval } \\
\text { (lower-upper } \\
\text { level) }\end{array}$ & $\begin{array}{l}\mathrm{p} \text {-Value } \\
\text { control } \\
\text { versus } \\
\text { probiotic }\end{array}$ & $\begin{array}{c}\text { Mastitis }^{\mathrm{a}} \\
\text { (control group, } \\
\mathrm{n}=49 ; \text { probiotic } \\
\text { group, } \mathrm{n}=26 \text { ) }\end{array}$ & $\begin{array}{c}95 \% \\
\text { Confidence } \\
\text { interval } \\
\text { (lower-upper } \\
\text { level) }\end{array}$ & $\begin{array}{l}\mathrm{p} \text {-Value } \\
\text { control } \\
\text { versus } \\
\text { probiotic }\end{array}$ & $\begin{array}{l}\mathrm{p} \text {-Value } \\
\text { healthy } \\
\text { versus } \\
\text { mastitis }\end{array}$ \\
\hline Staphylococcus & $\begin{array}{l}\text { Control } \\
\text { Probiotic }\end{array}$ & $\begin{array}{l}4.264 \pm 0.72 \\
3.983 \pm 0.69\end{array}$ & $\begin{array}{l}4.100-4.420 \\
3.831-4.138\end{array}$ & 0.013 & $\begin{array}{l}4.642 \pm 0.89 \\
4.270 \pm 0.57\end{array}$ & $\begin{array}{l}4.320-4.964 \\
4.025-4.515\end{array}$ & 0.065 & $\begin{array}{l}0.030 \\
0.080\end{array}$ \\
\hline Streptococcus & $\begin{array}{l}\text { Control } \\
\text { Probiotic }\end{array}$ & $\begin{array}{l}4.948 \pm 0.75 \\
4.765 \pm 0.65\end{array}$ & $\begin{array}{l}4.769-5.127 \\
4.616-4.929\end{array}$ & 0.147 & $\begin{array}{l}5.139 \pm 0.803 \\
5.501 \pm 0.65\end{array}$ & $\begin{array}{l}4.867-5.411 \\
4.788-5.313\end{array}$ & 0.647 & $\begin{array}{l}0.114 \\
0.068\end{array}$ \\
\hline Aerobes & $\begin{array}{l}\text { Control } \\
\text { Probiotic }\end{array}$ & $\begin{array}{l}4.314 \pm 1.34 \\
4.263 \pm 1.25\end{array}$ & $\begin{array}{l}4.073-4.560 \\
4.051-4.508\end{array}$ & 0.763 & $\begin{array}{l}4.209 \pm 1.19 \\
3.996 \pm 0.86\end{array}$ & $\begin{array}{l}3.763-4.654 \\
3.607-4.386\end{array}$ & 0.488 & $\begin{array}{l}0.637 \\
0.265\end{array}$ \\
\hline Anaerobes & $\begin{array}{l}\text { Control } \\
\text { Probiotic }\end{array}$ & $\begin{array}{l}4.205 \pm 1.25 \\
4.050 \pm 0.97\end{array}$ & $\begin{array}{l}3.994-4.434 \\
3.892-4.222\end{array}$ & 0.326 & $\begin{array}{l}4.266 \pm 1.20 \\
3.966 \pm 0.89\end{array}$ & $\begin{array}{l}3.820-4.712 \\
3.560-4.372\end{array}$ & 0.334 & $\begin{array}{l}0.975 \\
0.789\end{array}$ \\
\hline Lactobacillus & $\begin{array}{l}\text { Control } \\
\text { Probiotic }\end{array}$ & $\begin{array}{l}2.925 \pm 0.68 \\
3.050 \pm 0.64\end{array}$ & $\begin{array}{l}2.717-3.191 \\
2.869-3.300\end{array}$ & 0.486 & $\begin{array}{l}2.976 \pm 0.72 \\
2.846 \pm 0.17\end{array}$ & $\begin{array}{l}2.665-3.287 \\
2.746-2.946\end{array}$ & 0.529 & $\begin{array}{l}0.718 \\
0.202\end{array}$ \\
\hline IL-8 & $\begin{array}{l}\text { Control } \\
\text { Probiotic }\end{array}$ & $\begin{array}{l}1.84 \pm 0.42 \\
1.84 \pm 0.33\end{array}$ & $\begin{array}{l}1.757-1.910 \\
1.775-1.890\end{array}$ & 0.958 & $\begin{array}{l}2.437 \pm 0.46 \\
2.074 \pm 0.68\end{array}$ & $\begin{array}{l}2.177-2.697 \\
1.870-2.279\end{array}$ & 0.037 & $\begin{array}{r}<0.001 \\
0.034\end{array}$ \\
\hline
\end{tabular}

Bacterial counts showed as a mean of $\log _{10} \mathrm{CFU} / \mathrm{mL}$ milk \pm standard deviation. IL-8 showed as a mean of $\log _{10} \mathrm{pg} / \mathrm{mL}$ milk \pm standard deviation.

${ }^{a}$ Breast milk samples collected from mothers suffering from mastitis along the study.

IL, interleukin. 
probiotic group, but differences between groups were not statistically significant. Just a significant difference between groups is detected during the third month of intervention when percentage of analgesic treatment related to breast symptoms is lower in the probiotic group [11 women in the control group corresponding to $6.7 \%$ versus no treatment reported in the probiotic group during this period $(0 \% ; p=0.001)]$. The use of topic treatments for nipple cracks (pomade with lanoline) was mostly observed in the first month of breastfeeding $(4.7 \%$ of women used this kind of treatment). However the use of topical treatment was less frequent in the probiotic group [13 women in control group corresponding to $6 \%$ versus 4 women in probiotic group $(2 \% ; p=0.048)$.].

\section{Discussion}

Staphylococcus spp. is considered the most common etiological agent of mastitis. ${ }^{6,9,10}$ High counts of Staphylococcus spp. in breast milk are related to mastitis and painful breastfeeding. ${ }^{14,21}$ Previous studies have demonstrated the capability of L. fermentum CECT5716 to reduce Staphylococcus spp. load in breast milk of women suffering from mastitis and painful breastfeeding. ${ }^{13,14}$ The results of this study show that the consumption of L. fermentum CECT5716 during the breastfeeding period significantly reduces the incidence rate of mastitis by $51 \%$. In the control group, $24.6 \%$ of women suffered from mastitis. Data about incidence of mastitis are very variable. ${ }^{9}$ This variability is probably because of differences in diagnosis criteria. Mastitis incidence reported by studies using the same diagnosis criteria of L. fermentum consumption was slightly lower, between $13 \%$ and $20 \% .^{10,11,20}$ It has been previously reported that antibiotherapy during delivery was significantly more widely administered to women reporting mastitis (OR 1.53). ${ }^{22}$ These data might explain the slightly higher incidence observed in the L. fermentum study because women who received preventive antibiotic in the context of delivery were recruited. Regarding efficacy of probiotic strains to prevent mastitis, Fernández et al. ${ }^{11}$ evaluated the efficacy of a probiotic strain, Lactobacillus salivarius PS2, to prevent mastitis. They observed a similar decrease of mastitis incidence during the first 3 months of breastfeeding (56\%) for probiotic consumption. Although values of incidence reduction are similar to those of the L. fermentum study, there are important differences in protocols, apart from the different evaluated Lactobacillus species. For example, in the Fernández study, L. salivarius was administered during the last 10 weeks of pregnancy, but probiotic administration was not continued during breastfeeding. In the L. fermentum study, in which the probiotic strain was administered during breastfeeding, most episodes of mastitis occurred along the first 4 weeks postpartum. New studies should evaluate whether administration of L. fermentum during some weeks before childbirth might help to prevent more efficiently early episodes of mastitis.

Breast pain was quite frequent in women mainly during the first month, but in most cases it was not associated with clinical mastitis. L. fermentum consumption reduced the odds of suffering from pain and its intensity. In fact, in women who suffered from mastitis, the level of IL-8, a cytokine directly related to pain, ${ }^{23}$ was significantly lower in the probiotic group. Levels of IL-8 in breast milk were also directly re- lated to Staphylococcus load in milk. This relation was also observed in a previous study performed in women suffering from painful breastfeeding. ${ }^{14}$ IL- 8 is considered as an indicator of mastitis, ${ }^{9,24}$ so the reduction observed in the probiotic group in women suffering from mastitis is also indicative of lower level of inflammation and severity of mastitis.

Levels of Staphylococcus in milk were significantly lower in women receiving L. fermentum CECT5716. As high level of Staphylococcus is a risk factor for lactational mastitis, the reduction in this bacterial count might be responsible for the observed reduction in the incidence of mastitis. In fact, although higher level of Staphylococcus was found in milk of women who suffered from mastitis during the study, in the probiotic group this level tended to be lower.

The mechanism by which L. fermentum CECT5716 reduces the load of Staphylococcus in breast milk is not totally clear, although different activities of the probiotic strain might be involved. Antibacterial activity or competition phenomena have been described for L. fermentum CECT5716 in in vitro assays. ${ }^{16}$ However, although L. fermentum was detected in $61 \%$ more women in the probiotic group than women in the control group, we could not observe a significant presence of the strain in breast milk at the end of the intervention. Therefore, maybe local activity in the mammary gland is not mainly responsible for the activity. Other authors have suggested that metabolites with antibacterial properties produced by probiotic strains might reach the mammary gland and affect survival or virulence of Staphylococcus. ${ }^{11}$ L. fermentum showed in vitro the capability of inhibiting Staphylococcus growth in a diffusion agar assay, evidencing the production of antibacterial compounds that were not identified. ${ }^{16}$ In contrast, L. fermentum CECT5716 has shown immunoenhancing activity by increasing immunoglobulins and Th1 cytokines such as tumor necrosis factor (TNF)- $\alpha$ and IL-12. ${ }^{18}$ Staphylococcus spp. have a mechanism to avoid phagocytosis, the primary cellular defense of the mammary gland against pathogens. ${ }^{25}$ In animal models, L. fermentum CECT5716 increased the phagocytic activity of circulating blood leukocytes. This kind of activity in humans might help to prevent the proliferation of Staphylococcus $\mathrm{spp}$. in the mammary gland. ${ }^{17}$

A limitation of this study is the high level of dropouts during the study. Although women with firm intention of breastfeeding during the first 4 months were recruited, around $25 \%$ of women stop breastfeeding during the intervention. In Spain, where this study was performed, data of National Institute of Statistic estimated the prevalence of breastfeeding at the third month of lactation to be $53.5 \% .^{26}$ Most of the dropout cases took place during the first month of study. Women reported that they were too busy with their babies to commit to the study. Despite the number of women who complete the study was significantly lower than planned, the study keeps enough statistical power. It is because of a higher incidence of mastitis finally observed and the high difference in the level of incidence between groups.

The other limitation of the study is the population. To observe higher level of incidence, only women receiving preventive antibiotic were included. In another recent study performed with the other probiotic strain, L. salivarius PS2, population with history of mastitis was selected. ${ }^{11}$ In this 
study, the decrease of mastitis incidence in the probiotic group was similar to that observed in our study. Therefore, although more studies should be performed in the general population, the capability of certain probiotic strains to protect against mastitis does not seem to depend on these risk factors.

Besides being a randomized double-blinded trial controlled by placebo, another strength of this study is the measurement in the same study of the incidence of a disease, its risk factors, and parameters indicative of the severity of the disease.

Exclusive breastfeeding is recommended up to 6 months of age by the WHO because breast milk provides all the nutrients and components necessary for proper infant development and growth. ${ }^{27}$ Mastitis is an important health problem that affects a high percentage of women during lactation. This problem has a direct impact not only on the mothers threatening their health but also on the infants, as mastitis makes the normal breastfeeding difficult and in some cases can even lead to abandonment of breastfeeding. Otherwise, the symptoms of mastitis affect the mother-infant relationship because the symptoms of pain associated with mastitis might cause anxiety and feeling of anger because of the pain problems during breastfeeding. ${ }^{14}$ Until now, an appropriate management of breastfeeding is the recommendation for mastitis prevention. ${ }^{9}$ Results of this study show that L. fermentum CECT5716 consumption might be a new and efficient strategy to prevent the development of lactational mastitis.

\section{Acknowledgments}

MALDI-TOF analyses were possible with the help and collaboration of Dr. Federico García and the staff of the Department of Microbiology of the San Cecilio University Hospital, Granada, Spain. The authors wish to thank Llenalia García Fernández (SEPLIN, Soluciones Estadísticas Granada, Spain) for the collaboration of statistical analysis of the data in this study. This study was financed by the Andalusian Government and cofinanced by the European Regional Development Fund under the Andalusia's 2007-2013 Global Innovation-Technology-Enterprise Grant.

PROLAC Group members are: O. Bañuelos, Biosearch Life, Granada, Spain; S. Rite, Hospital U. Miguel Servet, Zaragoza, Spain; S. Luna, Hospital U. Virgen Macarena, Sevilla, Spain; M.C. Díaz-Faura, Hospital U. Virgen de la Arrixaca, Murcía, Spain; M.P. Ventura, Hospital C.U. Lozano Blesa, Zaragoza, Spain; L. Serrano-López, Hospital Materno-Infantil del CHU, Granada, Spain; M. Gil-Campos, Hospital Reina Sofia, Córdoba, Spain; E. Narbona, School of Medicine, University of Granada, Granada, Spain; C. Fuentes-Gutiérrez, Hospital G.U. Santa Lucía, Cartagena, Murcia, Spain; A. Iglesias-Deus, Hospital CHU, Santiago de Compostela, La Coruña, Spain; E.N. Rodilla, Hospital U., Salamanca, Spain; C. Rodríguez, Biosearch Life, Granada, Spain; A.D. Valero, Biosearch Life, Granada, Spain; and A. Sañudo, Biosearch Life, Granada, Spain.

\section{Disclosure Statement}

J.A.M.-L., M.P.D.-R., M.O., J.F., O.B., C.R., A.D.V., and A.S. are workers of Biosearch Life, owner of the patent of Lactobacillus fermentum CECT5716.

\section{References}

1. Schwiertz A. Microbiota of the human body: Implications in health and disease. Preface Adv Exp Med Biol 2016; 902: $\mathrm{V}$.

2. Sánchez B, Delgado S, Blanco-Míguez A, et al. Probiotics, gut microbiota, and their influence on host health and disease. Mol Nutr Food Res 2016; DOI: 10.1002/mnfr.201600240.

3. Vandenplas Y, Huys G, Daube G. Probiotics: An update. J Pediatr (Rio J) 2015;91:6-21.

4. Martín R, Langa S, Reviriego C, et al. Human milk is a source of lactic acid bacteria for the infant gut. $J$ Pediatr 2003;143:754-758.

5. Heikkilä MP, Saris PEJ. Inhibition of Staphylococcus aureus by the commensal bacteria of human milk. J Appl Microbiol 2003;95:471-478.

6. Delgado S, Arroyo R, Martín R, et al. PCR-DGGE assessment of the bacterial diversity of breast milk in women with lactational infectious mastitis. BMC Infect Dis 2008; 8:51.

7. Contreras GA, Rodríguez JM. Mastitis: Comparative etiology and epidemiology. J Mammary Gland Biol Neoplasia 2011;16:339-356.

8. Fernández L, Arroyo R, Espinosa I, et al. Probiotics for human lactational mastitis. Benef Microbes 2014;5:169183.

9. World Health Organization. Mastitis: Causes and Management. Geneva, Switzerland: World Health Organization, 2000.

10. Cullinane M, Amir LH, Donath SM, et al. Determinants of mastitis in women in the CASTLE study: A cohort study. BMC Fam Pract 2015;16:181.

11. Fernández L, Cárdenas N, Arroyo R, et al. Prevention of infectious mastitis by oral administration of Lactobacillus salivarius PS2 during late pregnancy. Clin Infect Dis 2016; 62:568-573.

12. Jiménez E, Fernández L, Maldonado A, et al. Oral administration of Lactobacillus strains isolated from breast milk as an alternative for the treatment of infectious mastitis during lactation. Appl Environ Microbiol 2008;74: 4650-4655.

13. Arroyo R, Martín V, Maldonado A, et al. Treatment of infectious mastitis during lactation: Antibiotics versus oral administration of Lactobacilli isolated from breast milk. Clin Infect Dis 2010;50:1551-1558.

14. Maldonado-Lobón JA, Díaz-López MA, Carputo R, et al. Lactobacillus fermentum CECT 5716 reduces Staphylococcus load in the breastmilk of lactating mothers suffering breast pain: A randomized controlled trial. Breastfeed Med 2015;10:425-432.

15. Martín R, Olivares M, Marín ML, et al. Probiotic potential of 3 lactobacilli strains isolated from human breast milk. J Hum Lact 2005;21:8-17.

16. Olivares M, Díaz-Ropero MP, Martín R, et al. Antimicrobial potential of four Lactobacillus strains isolated from breast milk. J Appl Microbiol 2006;101:72-79.

17. Díaz-Ropero MP, Martín R, Sierra S, et al. Two Lactobacillus strains, isolated from breast milk, differently modulate the immune response. J Appl Microbiol 2007;102: 337-343.

18. Olivares M, Díaz-Ropero MP, Sierra S, et al. Oral intake of Lactobacillus fermentum CECT5716 enhances the effects of influenza vaccination. Nutrition 2007;23:254-260.

19. Maldonado J, Cañabate F, Sempere L, et al. Human milk probiotic Lactobacillus fermentum CECT5716 reduces 
the incidence of gastrointestinal and respiratory infections in infants. J Pediatr Gastroenterol Nutr 2012;54: $55-61$.

20. Amir LH, Forster DA, Lumley J, et al. A descriptive study of mastitis in Australian breastfeeding women: Incidence and determinants. BMC Public Health 2007;7:62.

21. Delgado S, Arroyo R, Jiménez E, et al. Staphylococcus epidermidis strains isolated from breast milk of women suffering infectious mastitis: Potential virulence traits and resistance to antibiotics. BMC Microbiol 2009;9:82.

22. Mediano P, Fernández L, Rodríguez JM, et al. Case-control study of risk factors for infectious mastitis in Spanish breastfeeding women. BMC Pregnancy Childbirth 2014;14:195.

23. Cunha TM, Verri WA, Jr., Silva JS, et al. A cascade of cytokines mediates mechanical inflammatory hypernociception in mice. Proc Natl Acad Sci U S A 2005;102:17551760.

24. Hunt KM, Williams JE, Shafii B, et al. Mastitis is associated with increased free fatty acids, somatic cell count, and interleukin- 8 concentrations in human milk. Breastfeed Med 2013;8:105-110.
25. Avall-Jääskeläinen $S$, Koort $J$, Simojoki $H$, et al. Bovineassociated CNS species resist phagocytosis differently. BMC Vet Res 2013;9:227.

26. Informe anual del Sistema Nacional de Salud 2012. Edición revisada. Junio de 2015. Available at www.msssi.gob.es/ estadEstudios/estadisticas/sisInfSanSNS/tablasEstadisticas/ infsns2012.pdf (accessed October 2016).

27. Kramer MS, Kakuma R. The Optimal Duration of Exclusive Breastfeeding: A Systematic Review. Geneva, Switzerland: World Health Organization, 2002.

Address correspondence to: Juristo Fonollá, PhD Research Department Biosearch Life Camino de Purchil 66 18004 Granada Spain

E-mail: juristo.fonollajoya@biosearchlife.com 